Sharif University of Technology
Scientia Iranica
Transactions E: Industrial Engineering
SCIENTIA
I RAN I C A

\title{
Selecting unique suppliers through winner determination in combinatorial reverse auction: Scatter search algorithm
}

\author{
R. Alaei and M. Setak* \\ Faculty of Industrial Engineering, K.N. Toosi University of Technology, Tehran, Iran. \\ Received 16 September 2015; received in revised form 13 July 2016; accepted 8 October 2016
}

KEYWORDS
Outsourcing;
Supplier selection;
Combinatorial reverse
auction;
Winner determination
problem;
Scatter search;
Taguchi method.

\section{Introduction}

Outsourcing is the action or practice of obtaining required items (goods or services) by contract from outside sources, and it helps companies to perform well in their core competencies and reduce lack of skill or expertise in areas where they want to outsource [1]. Supplier selection is one of the critical phases of outsourcing because a selected supplier will be a close associate of the company for a considerable period of time, during which the two parties will be

\footnotetext{
*. Corresponding author. Tel.: +982184063373; Fax: +982188674858

E-mail addresses: ralaei@mail.kntu.ac.ir (R. Alaei); setak@kntu.ac.ir (M. Setak)
}

forced to cooperate and support each other in good and bad times [2]. Selecting suppliers can be viewed as an allocation problem in which a set of potential suppliers is evaluated in terms of some quantitative and qualitative criteria, and the most efficient set of suppliers among potential suppliers is determined to assign the task of supplying required items $[3,4]$.

Auctions are used as popular ways for allocating items or tasks to multiple agents to maximize revenue or minimize cost. Single-item auctions, such as English and Vickrey auctions, are the most common auction formats, but they are not always efficient [5]. Combinatorial auction, as one of multi-item auction formats, enables bidders to place all-or-nothing bids on any subset of items (i.e., bundles of items) rather than just on individual items according to their personal preferences. Combinatorial auctions are efficient when bidders are interested in multiple items 
and their valuations for these items are non-additive, particularly when complementary relationships exist among items. For this reason, they have attracted considerable attention in the auction literature that is reviewed by Abrache et al. [6], Blumrosen and Nisan [7], Bichler et al. [8], and Hoffman [9], to name a few. Combinatorial auctions have various applications, such as auctioning airport time slots and resources [10], truckload transportation [11], bus routes [12], advertising time slots [13,14], spectrum licenses [15], and timber allocation [16]. Some researchers have reported that applying combinatorial auctions to companies' procurement processes can lead to significant savings. Bichler et al. [8] and Hohner et al. [17] studied the use of combinatorial reverse auction in sourcing process of Mars Incorporated in its webpage designed by MarsIBM team, and they reported significant savings in Mars's procurement costs. Metty et al. [18] studied the Motorola Company's reinvention in its supplier negotiation process which uses an advanced Internet-based negotiation platform for sourcing, and they reported a reduction in the required time and effort for negotiation and significant savings in procurement costs (\%3.75, about $\$ 600$ million in 2005) due to using combinatorial reverse auction in supplier negotiation process. Also, Sandholm et al. [19] studied the changed approach of Procter \& Gamble (P\&G) in sourcing which puts into practice CombineNet's approach in building sourcing networks by using combinatorial reverse auction in its sourcing processes. They reported significant savings in sourcing costs for P\&G $(\% 9.6$, about $\$ 295$ million over a period of two and a half years until March in 2005) as well as a reduction in the required time for sourcing processes from months to weeks.

In a procurement scenario, there is a buyer who wants to buy a set of items efficiently in terms of some special criteria and a set of potential suppliers who can supply the required items. The buyer can hold a reverse auction to buy the required items. If there are complementary relationships between tasks of supplying some tendered items (i.e., synergies in terms of supplying cost), a combinatorial reverse auction can be beneficial in which potential suppliers can express their preferences and submit several bids on combinations of those items that can result in significant savings in procurement costs of companies [8].

In this paper, the problem of selecting suppliers for the required items of a company is considered in which there are complementary relationships between tasks of supplying some required items due to economies of scale in their supplying. Therefore, we propose a combinatorial reverse auction mechanism to select the most efficient set of suppliers among a set of potential suppliers. It is assumed that the task of supplying each required item is indivisible to multiple suppliers, or the company prefers to select only one supplier for supplying each required item. So, the winner determination in our proposed combinatorial reverse auction mechanism is done in such a way that supplying each tendered item is assigned to only one potential supplier. This is the main difference of our research with similar studies in existing literature, such as the research papers of Hsieh [5], Bichler et al. [8], Sandholm et al. [19], and Olivares et al. [20], which have used the combinatorial reverse auction for selecting suppliers. We formulate the corresponding winner determination problem of the proposed combinatorial reverse auction mechanism as a binary integer program with the objective of minimizing the company's procurement costs. The formulated winner determination problem is an NP-complete combinatorial optimization problem. So, the time required to solve this problem, using any currently known algorithm, increases exponentially as the size of the problem grows [21]. Therefore, exact methods will fail to solve the largescale instances of formulated winner determination problem in a reasonable time. So, we propose a metaheuristic algorithm called scatter search for finding its feasible and near-optimal solutions.

The rest of the paper is organized as follows. In Section 2, our proposed combinatorial reverse auction mechanism is described for selecting the most efficient set of suppliers among a set of potential suppliers. Section 3 formulates the corresponding winner determination problem of the proposed combinatorial reverse auction mechanism as a single-objective binary integer program. In Section 4, a problem-specific scatter search algorithm is proposed to solve the formulated winner determination problem. Section 5 presents the computational results and the performance of the proposed scatter search algorithm evaluated by solving several randomly generated instances of winner determination problem with different real-world sizes. Finally, in Section 6, conclusions and future research directions are summarized.

\section{Combinatorial reverse auction mechanism}

Suppose that a company has decided to supply some of its required items (goods or services) from external sources. It is assumed that there are complementary relationships between tasks of supplying some required items due to economies of scale in their supplying. The complementary relationship between supplying two items means that the supplying cost of items together is less than the sum of their individual supplying costs. So, to select the most efficient suppliers among a set of potential suppliers, the company conducts a combinatorial reverse auction. As mentioned in the previous section, the combinatorial reverse auction establishes an environment in which potential suppliers can compete and express their preferences about tendered items 
we can easily say that the formulated winner determination problem is NP-hard. But, Sandholm et al. [24] proved that the winner determination problem of single-unit combinatorial reverse auction with/without free disposal is NP-complete. Also, they proved that finding feasible solutions to winner determination problem of single-unit combinatorial reverse auction without free disposal by considering XOR constraints (i.e., formulated winner determination problem) is NPcomplete. Therefore, finding feasible solutions to the formulated winner determination problem as well as its solving is NP-complete. Although any given solution to an NP-complete problem can be verified quickly (in polynomial time), there is no known efficient way to locate a solution in the first place. Indeed, the most notable characteristic of NP-complete problems is that no fast solution to them is known. That is, the time required to solve the problem using any currently known algorithm increases exponentially as the size of the problem grows [21].

Therefore, exact methods will fail to solve the large-scale instances of formulated winner determination problem in a reasonable time. So, we propose a meta-heuristic algorithm called scatter search to find its feasible and near-optimal solutions.

\section{Scatter search}

Scatter search is a population-based meta-heuristic which exploits the knowledge of the problem to create new and thus better solutions from the combination of existing ones. The fact that the relevant information regarding the optimal solution is embedded in a diversified subset of elite solutions is one of the fundamentals of scatter search. Scatter search takes multiple solutions into account as a foundation for creating new ones, and it uses heuristics which combine them through mechanisms that promote diversity and quality. So, scatter search enhances the exploration of the information not contained in each solution individually. The usual process for solving a problem by means of creating progressively better solutions is divided into the following components [25-27]:

- Diversification generation method: Creates a collection of trial solutions;

- Improvement method: Transforms the trial solutions into enhanced ones and usually restores feasibility;

- Reference set update method: Maintains the reference set with the best solutions according to certain criteria;

- Subset generation method: Creates subsets of solutions from the reference set;

- Solution combination method: Combines solutions from each subset, thus creating new ones.
In what follows, the proposed problem-specific scatter search method that is used to find feasible and near-optimal solutions to winner determination problem is explained in detail.

\subsection{Representation of solutions}

Before explaining the details of solution procedure, the scheme that is used to represent the solutions in the search space is described. A solution in the search space is represented with $Y=\left(y_{1}, y_{2}, \cdots, y_{n}\right)$ in which $y_{j} \in Z K_{j}=\{0\} \cup K_{j}$. A non-zero value for $y_{j}$ means that potential supplier $j$ is one of the winners of combinatorial reverse auction, and the value of $y_{j}$ represents the index of his accepted bid. With this representation scheme, satisfaction of XOR constraints is ensured.

\subsection{Generating a population of diverse solutions}

For creating a population, $P O P$, including $N$ nonduplicate solutions, a random generation method is used. Solution $Y$ in $P O P$ is constructed by generating $y_{j}$ as a uniformly distributed random number in $Z K_{j}$, for all $j \in J$. Since finding a feasible solution to the formulated winner determination problem is NPcomplete, infeasible solutions are allowed to enter the population of solutions.

\subsection{Improving the solutions in population}

Since infeasible solutions allow entering the population of solutions, we use an improvement procedure to adjust the solutions in population with the aim of decreasing their infeasibility. For this purpose, we first define the left-hand side value of $i$ th equality constraint for solution $Y$ as follows:

$$
\operatorname{LHS}_{i}(Y)=\sum_{j \in J \mid y_{j} \neq 0} a_{i j, y_{j}} \quad \forall i \in I .
$$

Also, we define $\delta_{i}(Y)$ as inclusion state of $i$ th tendered item in at least one accepted bid of solution $Y$ :

$$
\delta_{i}(Y)=\left\{\begin{array}{ll}
1 & \operatorname{LHS}_{i}(Y) \geq 1 \\
0 & \operatorname{LHS}_{i}(Y)=0
\end{array} \quad \forall i \in I .\right.
$$

Then, we use the following necessary conditions for solution $Y$ to determine if it is a candidate of improvement or not:

(a) $\sum_{i \in I} \delta_{i}(Y)=m$

(b) $\exists i \in I, \operatorname{LHS}_{i}(Y)>1$.

The first condition (a) means that all tendered items should be included in accepted bids of solution $Y$, and the second condition (b) means that there should be at least one tendered item that is included more than one time in accepted bids of solution $Y$. Therefore, all 


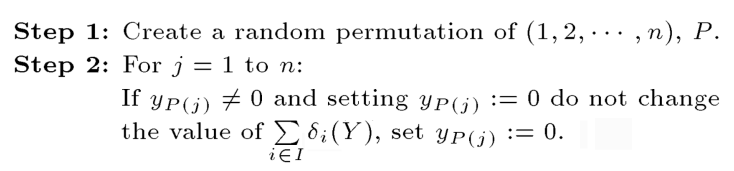

Figure 1. Improving solutions in population.

solutions in population are checked and if a solution has these conditions, the improvement procedure that is described in Figure 1 is run $M$ times for decreasing its infeasibility. A solution that has these conditions may be improved using this procedure, and if the result is a non-duplicate solution, it is added to the population of solutions, i.e., POP.

\subsection{Evaluation of solutions}

To evaluate the solutions in population, two criteria are used. The first criterion is the value of objective function, i.e. the total procurement cost of tendered items. This criterion for solution $Y$ is calculated as follows:

$$
\operatorname{Cost}(Y)=\sum_{j \in J \mid y_{j} \neq 0} c_{j, y_{j}} .
$$

Furthermore, since infeasible solutions are allowed to enter the population of solutions, infeasibility is considered as the second criterion which is defined for solution $Y$ as follows:

$$
\operatorname{Inf}(Y)= \begin{cases}\sum_{i \in I} \alpha_{i}(Y)+\frac{\sum_{i \in I} \beta_{i}(Y)}{\sum_{i \in I} \alpha_{i}(Y)} & \sum_{i \in I} \alpha_{i}(Y)>0 \\ 0 & \sum_{i \in I} \alpha_{i}(Y)=0\end{cases}
$$

in which $\alpha_{i}(Y)$ and $\beta_{i}(Y)$ are defined as violation state and violation severity of $i$ th equality constraint, respectively:

$$
\begin{aligned}
& \alpha_{i}(Y)=\left\{\begin{array}{ll}
1 & \operatorname{LHS}_{i}(Y) \neq 1 \\
0 & \operatorname{LHS}_{i}(Y)=1
\end{array} \quad \forall i \in I,\right.
\end{aligned} \quad \begin{aligned}
& \beta_{i}(Y)= \begin{cases}\frac{\operatorname{LHS}_{i}(Y)-1}{\sum_{j \in J} \sum_{k \in K_{j}} a_{i j k}-1} & \operatorname{LHS}_{i}(Y) \geq 1 \\
1 & \operatorname{LHS}_{i}(Y)=0\end{cases}
\end{aligned}
$$

\subsection{Building reference set}

After improving a population of solutions, a reference set, including $B$ solutions, from the population is selected for combining and creating new solutions. The reference set, RefSet, has two parts: RefSet ${ }_{1}$

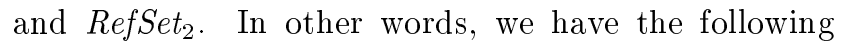
equation:

$$
\text { RefSet }=\text { RefSet }_{1} \cup \text { RefSet }_{2} .
$$

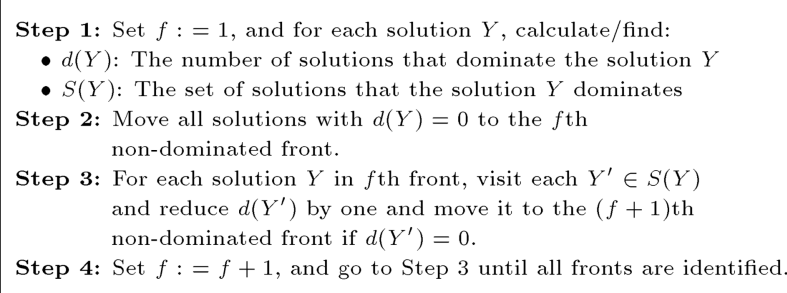

Figure 2. Non-dominated sorting of solutions in population.

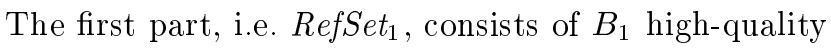
solutions from the population. The criterion for select-

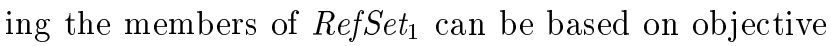
function value or infeasibility of solutions. The difficulty with these criteria for a minimization problem is that the solutions with less objective function value generally have higher infeasibility, and vice versa. Thus, if the selection is favored on the solutions with less objective function value, choosing solutions, mostly infeasible, is likely. This will not help scatter search in finding feasible solutions to winner determination problem. On the other hand, if the selection is favored on less infeasible solutions, selecting solutions with higher objective function value is likely. Therefore, selection should be done in such a way that both of these criteria are improved. To do this, the solutions in population are sorted based on these criteria using a fast non-dominated sorting procedure proposed by Deb et al. [28] that is described in Figure 2. It should be noted that solution $Y$ dominates solution $Y^{\prime}$ if:

$$
[\operatorname{Cost}(Y), \operatorname{In} f(Y)] \neq\left[\operatorname{Cost}\left(Y^{\prime}\right), \operatorname{In} f\left(Y^{\prime}\right)\right]
$$

and:

$$
[\operatorname{Cost}(Y), \operatorname{Inf}(Y)] \leq\left[\operatorname{Cost}\left(Y^{\prime}\right), \operatorname{In} f\left(Y^{\prime}\right)\right] .
$$

The non-dominated sorting procedure ranks the solutions in different non-dominated fronts, and then $B_{1}$ solutions from the best non-dominated fronts are selected as members of RefSet ${ }_{1}$. The second part of reference set, i.e. RefSet 2 , includes $B_{2}$ diverse solutions from the remained population, i.e. $P O P \backslash$ RefSet. To select the first member of RefSet ${ }_{2}$, for all $Y \in P O P \backslash$ RefSet, the minimum distance of solution $Y$ from the members of RefSet is calculated as follows:

$$
D(Y, \text { RefSet })=\min _{Y^{\prime} \in \operatorname{RefSet}}\left(\sum_{j \in J} D_{j}\left(Y, Y^{\prime}\right)\right),
$$

in which:

$$
D_{j}\left(Y, Y^{\prime}\right)=\left\{\begin{array}{ll}
1 & y_{j} \neq y_{j}^{\prime} \\
0 & y_{j}=y_{j}^{\prime}
\end{array} \quad \forall j \in J .\right.
$$

Then, solution $\hat{Y}$ that is determined by Eq. (14) is 
removed from POP $\backslash$ RefSet and added to RefSet ${ }_{2}$. This process is repeated $B_{2}$ times by updating the value of $D(Y$, RefSet $)$ for all $Y \in P O P \backslash$ RefSet:

$$
\hat{Y}=\underset{Y \in P O P \backslash \text { RefSet }}{\arg \max } D(Y, \text { RefSet }) .
$$

\subsection{Combining solutions in reference set}

To create new non-duplicate solutions, NewYs, all pairs of solutions in the reference set are combined. So, the number of new solutions created in this phase is equal to $B(B-1) / 2$. Let $Y^{\prime}$ and $Y^{\prime \prime}$ be two members of the reference set. To create new solution $Y$, a uniformly distributed random number in $[0,1], r_{j}$, is generated for all $j \in J$ and the value of $y_{j}$ is determined as follows:

$$
y_{j}=\left\{\begin{array}{ll}
y_{j}^{\prime} & r_{j} \in[0,0.4] \\
y_{j}^{\prime \prime} & r_{j} \in(0.4,0.8] \\
0 & r_{j} \in(0.8,0.9] \\
\operatorname{Bid}_{j} & r_{j} \in(0.9,1]
\end{array} \quad \forall j \in J,\right.
$$

where $B i d_{j}$ is a random number in $Z K_{j}$ that is calculated as a function of $r_{j}$ and $b_{j}$ :

$$
\operatorname{Bid}_{j}=\left\lceil\frac{r_{j}-0.9}{1-0.9} \times b_{j}\right\rceil \quad \forall j \in J .
$$

Example 1. Suppose that:

$$
\begin{aligned}
& n=10, \\
& \mathbf{b}=(5,6,5,7,4,5,9,5,5,8), \\
& Y^{\prime}=(0,5,3,0,1,1,2,0,4,7), \\
& Y^{\prime \prime}=(5,1,4,5,0,0,0,3,2,0) .
\end{aligned}
$$

New solution $Y=(0,1,4,0,0,5,0,3,0,7)$ is created by combining $Y^{\prime}$ and $Y^{\prime \prime}$ as illustrated in Figure 3.

\subsection{Updating the reference set}

After combining the solutions in the reference set and creating new solutions, RefSet $\cup N e w Y s$ is considered as the population of solutions and the improvement procedure is applied to its members. Then, the objective function value and infeasibility of solutions in improved population are evaluated and the new reference set is constructed using the reference set building method, explained in Subsection 4.5.

\begin{tabular}{ccccc|c|c|c|c|c|c|c|}
$b_{j}$ & 5 & 6 & 5 & 7 & 4 & 5 & 9 & 5 & 5 & 8 \\
$Y^{\prime}$ & 0 & 5 & 3 & 0 & 1 & 1 & 2 & 0 & 4 & 7 \\
$Y^{\prime \prime}$ & 5 & 1 & 4 & 5 & 0 & 0 & 0 & 3 & 2 & 0 \\
$r_{j}$ & 0.14 & 0.91 & 0.54 & 0.23 & 0.79 & 0.99 & 0.47 & 0.66 & 0.84 & 0.39 \\
$Y$ & 0 & 1 & 4 & 0 & 0 & 5 & 0 & 3 & 0 & 7 \\
\hline
\end{tabular}

Figure 3. Combining two solutions to create a new one.

\subsection{Stopping condition}

Creating new solutions and updating the reference set continue if at least one better solution is found in each iteration, or else another population, including solutions in RefSet ${ }_{1}$ and $N-B / 2$ numbers of randomly generated diverse solutions, is used to continue the search procedure. If the number of iterations is equal to a given number, $T$, the scatter search stops and the results are reported.

\subsection{Outline of scatter search procedure}

The outline of the proposed scatter search method for finding feasible and near-optimal solutions of winner determination problem in the combinatorial reverse auction is described in Figure 4.

\section{Computational experiments}

To evaluate the performance of the proposed scatter search method in finding feasible and near-optimal solutions to winner determination problem, it is tested with several randomly generated problem instances with different sizes. The size of a problem instance is determined by the number of tendered items $(m)$, the number of potential suppliers $(n)$, and the number of their bids $\left(b_{j}\right)$. In Table 1 , we have listed different sizes of problem instances used to evaluate the performance of the proposed scatter search algorithm. The problem instance generation procedure in the Appendix is used to generate 3 instances for each problem size.

\subsection{Tuning parameters}

Meta-heuristics show different performances when various values of their parameters are used. Therefore, the use of a calibration method is necessary to achieve better performances. Taguchi has developed a fractional factorial design based on different factors and their levels that uses a reduced number of experiments. This method can be used for tuning the parameters of metaheuristics (e.g., see $[29,30])$. Taguchi has classified the factors affecting a response into two categories, including controllable factors and noise factors. Considering orthogonal arrays, he has suggested a method to reduce the change around the target so that the best design is the one that is impressed less by noise factors. In the Taguchi method, there are two approaches to analyze the results. The first one is the analysis of variance that is applied to experiments with one replication, and the

Table 1. Different sizes of problem instances.

\begin{tabular}{cccc}
\hline Size \# & $\boldsymbol{m}$ & $\boldsymbol{n}$ & $\boldsymbol{b}_{\boldsymbol{j}}$ \\
\hline I & 10 & 25 & $\leq 10$ \\
II & 10 & 50 & $\leq 10$ \\
III & 20 & 50 & $\leq 10$ \\
IV & 20 & 75 & $\leq 10$ \\
\hline
\end{tabular}




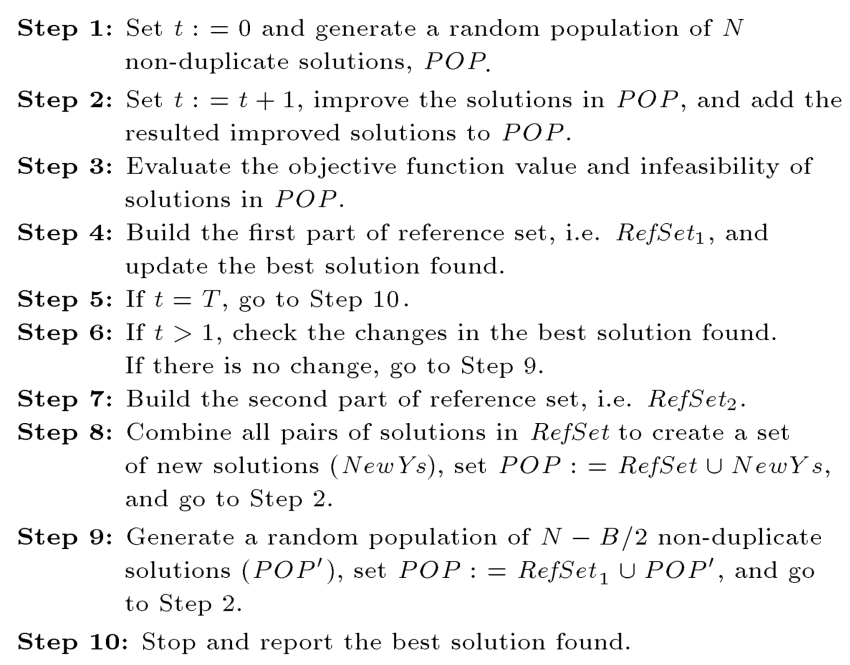

Step 10: Stop and report the best solution found.

Figure 4. Outline of the proposed scatter search procedure.

second one is the signal-to-noise ratio analysis which is suggested for experiments with multiple runs [31]. Since meta-heuristics run several times in order to achieve high-quality solutions to problems, the signalto-noise ratio analysis is used here to analyze the results and tune the parameters of proposed scatter search method. To do this, first, the parameters (factors) that affect the performance of scatter search method as well as their values (levels) are determined in Table 2. Note that the values of parameters in Taguchi method are defined based on a trial and error procedure on a problem instance [31]. Here, the first instance in Table A.1 (in Appendix), i.e. I-1, is used for defining the values of scatter search parameters. The aim of the Taguchi method for a minimization problem is to find a combination of parameters' values such that the signal-to-noise ratio is maximized. The signal-to-noise ratio is defined as follows in which $z_{r}$ is the target (response) in the Taguchi method and $R$ is the number of replications [31]. Here, the response is the objective function value of winner determination problem:

$$
\mathrm{SN}=-10 \log \left(\frac{1}{R} \sum_{r=1}^{R} z_{r}^{2}\right)
$$

Table 2. Parameters of scatter search method and their values.

\begin{tabular}{ccccc}
\hline Parameters & \multicolumn{4}{c}{ Values } \\
\hline $\boldsymbol{N}$ & 50 & 100 & 150 & 200 \\
$\boldsymbol{B}$ & 10 & 20 & & \\
$\boldsymbol{M}$ & $m / 2$ & $m$ & $3 m / 2$ & $2 m$ \\
\hline
\end{tabular}

The Taguchi method for tuning the parameters of scatter search is implemented in Minitab ${ }^{\circledR} 17.1$. Assuming that $T=100$ and $R=30$, the experimental results of scatter search method using $L^{16}$ orthogonal arrays from the Taguchi method for the first instance of each problem size, i.e., I-1, II-1, III-1, and IV-1, are shown in Table 3. Based on the information in Table 3, the mean of signal-to-noise ratios for different values of parameters is calculated and listed in Table 4. Now, the best values of parameters that maximize the mean of signal-to-noise ratios can be determined for solving different problem sizes. These values are listed in Table 5 .

\subsection{Results and comparison}

The proposed scatter search method, with $T=2 \mathrm{mn}$ and the best values of parameters, is run 10 times for each problem instance using MATLAB ${ }^{\circledR} 7.6$ in a computer with Intel ${ }^{\circledR}$ Core $^{\mathrm{TM}}$ i $5(2.53 \mathrm{GHz}) \mathrm{CPU}$ and 4 GB RAM. Computational results of different problem instances, including the best feasible solution found by scatter search and its average runtime as well as the results of using $\operatorname{LINGO}^{\circledR} 8.0$ for solving the problem instances, are summarized in Table 6. In Figure 5, performance of the proposed scatter search in solving different instances of winner determination problem is compared with LINGO $^{\circledR}$ software. As illustrated in Figure 5, the proposed scatter search performs better than the used software in finding feasible and nearoptimal solutions to the problem instances with larger sizes. Also, Figure 6 compares the average runtime of the proposed scatter search method with LINGO $^{\circledR}$ software and shows a significant difference between their average runtime in solving different instances of winner determination problem, especially instances with larger sizes. 
Table 3. Experimental results of scatter search using Taguchi method for different problem sizes.

\begin{tabular}{cccccccc}
\hline \multicolumn{2}{c}{ Parameters $\&$ values } & & \multicolumn{4}{c}{ Signal to noise ratios } \\
\cline { 1 - 2 } \cline { 5 - 7 } $\boldsymbol{N}$ & $\boldsymbol{B}$ & $\boldsymbol{M}$ & & I-1 & II-1 & III-1 & IV-1 \\
\hline 50 & 10 & $m / 2$ & & -53.6768 & -55.1309 & -68.2404 & -67.4761 \\
50 & 10 & $m$ & & -53.6204 & -55.0990 & -68.2435 & -67.5454 \\
50 & 20 & $3 m / 2$ & & -53.5930 & -54.7381 & -67.6144 & -67.3838 \\
50 & 20 & $2 m$ & & -53.5664 & -54.7376 & -67.7082 & -67.3549 \\
100 & 10 & $m / 2$ & & -53.6497 & -55.0612 & -67.9354 & -67.4732 \\
100 & 10 & $m$ & & -53.6238 & -54.9884 & -67.8690 & -67.4302 \\
100 & 20 & $3 m / 2$ & & -53.5889 & -54.6328 & -67.7745 & -67.1794 \\
100 & 20 & $2 m$ & & -53.5741 & -54.7128 & -67.7235 & -67.1636 \\
150 & 20 & $m / 2$ & & -53.5611 & -54.6501 & -67.6017 & -67.2584 \\
150 & 20 & $m$ & & -53.5723 & -54.7387 & -67.6783 & -67.2087 \\
150 & 10 & $3 m / 2$ & & -53.5976 & -54.9599 & -67.9714 & -67.3310 \\
150 & 10 & $2 m$ & & -53.5902 & -55.0376 & -67.9243 & -67.3843 \\
200 & 20 & $m / 2$ & & -53.5604 & -54.7261 & -67.6029 & -67.1597 \\
200 & 20 & $m$ & & -53.5832 & -54.6916 & -67.6354 & -67.1643 \\
200 & 10 & $3 m / 2$ & -53.5623 & -54.9610 & -68.0290 & -67.3077 \\
200 & 10 & $2 m$ & -53.5980 & -55.0080 & -67.9769 & -67.2995 \\
\hline
\end{tabular}

Table 4. Mean of signal-to-noise ratios for different problem sizes.

\begin{tabular}{|c|c|c|c|c|c|}
\hline \multirow{2}{*}{ Parameters } & \multirow{2}{*}{ Values } & \multicolumn{4}{|c|}{ Mean of SN ratios } \\
\hline & & I-1 & II-1 & III-1 & IV-1 \\
\hline \multirow{4}{*}{$N$} & 50 & -53.614 & -54.926 & -67.952 & -67.440 \\
\hline & 100 & -53.609 & -54.849 & -67.826 & -67.312 \\
\hline & 150 & -53.580 & -54.846 & -67.794 & -67.296 \\
\hline & 200 & $\underline{-53.576}$ & -54.847 & -67.811 & -67.233 \\
\hline \multirow{2}{*}{$B$} & 10 & -53.615 & -55.031 & -68.024 & -67.406 \\
\hline & 20 & -53.575 & -54.703 & -67.667 & -67.234 \\
\hline \multirow{4}{*}{$M$} & $m / 2$ & -53.612 & -54.892 & -67.845 & -67.342 \\
\hline & $m$ & -53.600 & -54.879 & -67.857 & -67.337 \\
\hline & $3 m / 2$ & -53.585 & -54.823 & -67.847 & -67.300 \\
\hline & $2 m$ & -53.582 & -54.874 & -67.833 & -67.301 \\
\hline
\end{tabular}

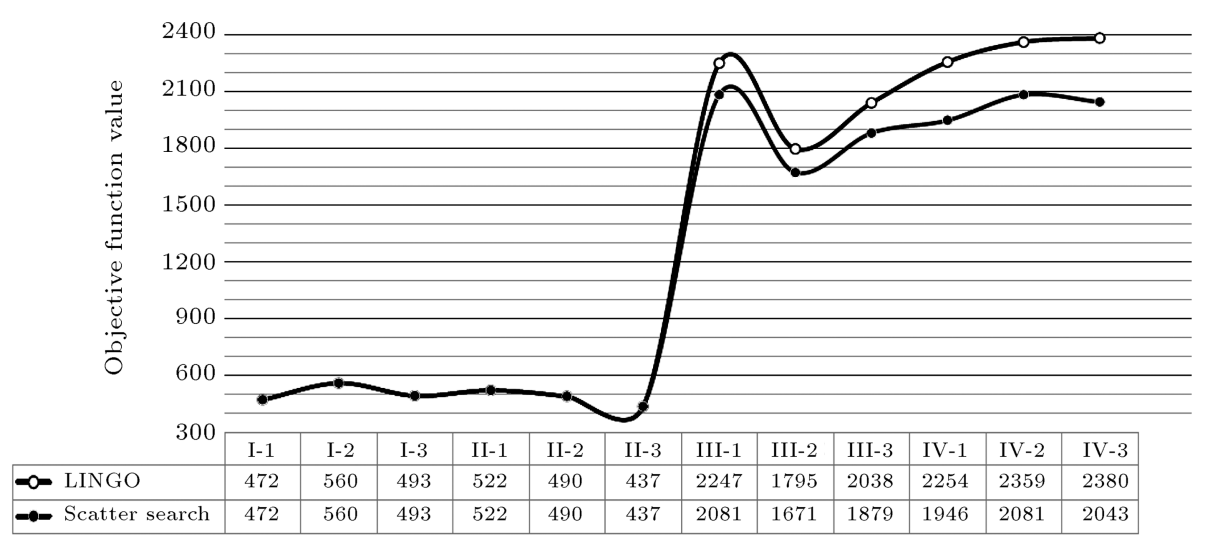

Figure 5. Comparing the performances of scatter search and $\mathrm{LINGO}^{\circledR}$. 
Table 5. The best values of scatter search parameters for different problem sizes.

\begin{tabular}{ccccc}
\hline \multirow{2}{*}{ Parameters } & \multicolumn{4}{c}{ Best values } \\
\cline { 2 - 5 } & I & II & III & IV \\
\hline $\boldsymbol{N}$ & 200 & 150 & 150 & 200 \\
$\boldsymbol{B}$ & 20 & 20 & 20 & 20 \\
$\boldsymbol{M}$ & 20 & 15 & 40 & 30 \\
\hline
\end{tabular}

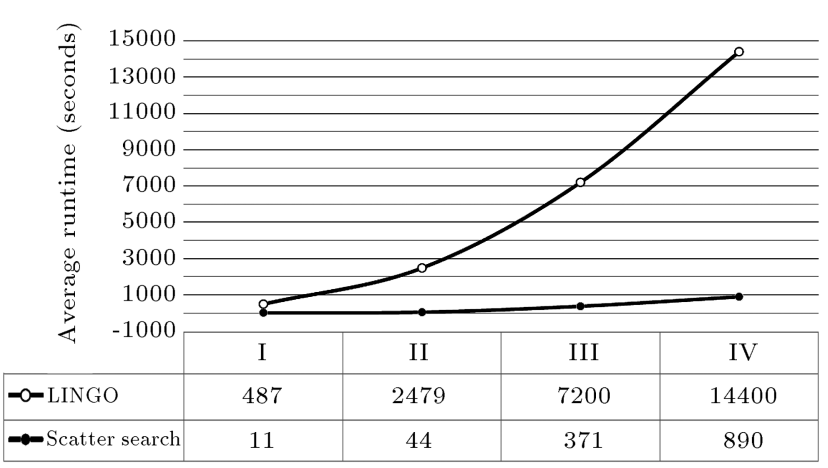

Figure 6. Comparing the average runtime of scatter search and $\mathrm{LINGO}^{\circledR}$.

\section{Conclusions}

In this research, we proposed a combinatorial reverse auction mechanism for selecting the most efficient suppliers for required items of a company. As a contribution, it was assumed that the task of supplying each required item is indivisible to multiple suppliers, or the company prefers to select only one supplier for supplying each required item. So, the winner determination process was done in such a way that supplying each tendered item is assigned to only one potential supplier. Development of a meta-heuristic algorithm called scatter search with problem-specific components for solving the corresponding winner determination problem was another contribution in this research. The experimental results of evaluating the performance of the proposed algorithm show that the proposed scatter search with parameters tuned by Taguchi method performs well in solving different sizes of problem instances with respect to solution quality and runtime. Some of directions for the future research are as follows:

- Formulating the winner determination problem with either more or other decision criteria such as quality and delivery time;

- Formulating the winner determination problem by introducing other constraints, e.g., lower and upper bounds for the number of winners (i.e., selected suppliers);

- Developing other meta-heuristic algorithms to target the resulted winner determination problems;

- Developing exact methods for solving the resulted winner determination problems and using the output of meta-heuristics as the initial solutions for exact methods;

- Applying the proposed combinatorial reverse auction mechanism in selecting unique suppliers for providing the following services in multiple regions of an electric power distribution company's responsibility area:

- Repairing some or all parts of distribution network including distribution lines and substations;

Table 6. Computational results for different problem instances.

\begin{tabular}{|c|c|c|c|c|c|c|}
\hline \multirow{2}{*}{ Instance \# } & \multicolumn{3}{|c|}{ LINGO $^{\circledR}$} & \multicolumn{3}{|c|}{ Scatter search } \\
\hline & Obj. value & Optimal & Runtime & Best obj. value & Optimal & Average runtime \\
\hline I-1 & 472 & Yes & 528 & 472 & Yes & 9 \\
\hline $\mathrm{I}-2$ & 560 & Yes & 436 & 560 & Yes & 12 \\
\hline $\mathrm{I}-3$ & 493 & Yes & 496 & 493 & Yes & 11 \\
\hline II-1 & 522 & Yes & 2431 & 522 & Yes & 44 \\
\hline II-2 & 490 & Yes & 2380 & 490 & Yes & 41 \\
\hline II-3 & 437 & Yes & 2626 & 437 & Yes & 47 \\
\hline III-1 & 2247 & No & 7200 & 2081 & Unknown & 371 \\
\hline III-2 & 1795 & No & 7200 & 1671 & Unknown & 348 \\
\hline III-3 & 2038 & No & 7200 & 1879 & Unknown & 395 \\
\hline IV-1 & 2254 & No & 14400 & 1946 & Unknown & 887 \\
\hline IV -2 & 2359 & No & 14400 & 2081 & Unknown & 894 \\
\hline IV-3 & 2380 & No & 14400 & 2043 & Unknown & 890 \\
\hline
\end{tabular}


- Repairing some or all types of sustained power interruptions that occur in distribution network.

\section{References}

1. Gunasekaran, A., Irani, Z., Choy, K.L., Filippi L. and Papadopoulos, T. "Performance measures and metrics in outsourcing decisions: a review for research and applications", Int. J. of Prod. Econ., 161, pp. 153-166 (2015).

2. Vaxevanou, A. and Konstantopoulos, N. "Basic principles and the philosophy of outsourcing", Procedia Social and Behav. Sci., 175, pp. 567-571 (2015).

3. Setak, M., Sharifi, S. and Alimohammadian, A. "Supplier selection and order allocation models in supply chain management: a review", World Appl. Sci. J., 18(1), pp. 55-72 (2012).

4. Soroor, J., Tarokh, M.J., Khoshalhan, F. and Sajjadi, S. "Intelligent evaluation of supplier bids using a hybrid technique in distributed supply chains", J. of Manuf. Syst., 31(2), pp. 240-252 (2012).

5. Hsieh, F. "Combinatorial reverse auction based on revelation of Lagrangian multipliers", Decis. Support Syst., 48(2), pp. 323-330 (2010).

6. Abrache, J., Crainic, T.G., Gendreau, M. and Rekik, M. "Combinatorial auctions", Ann. of Oper. Res., 153(1), pp. 131-164 (2007).

7. Blumrosen, L. and Nisan, N. "Combinatorial auctions", In Algorithmic Game Theory, N. Nisan, T. Roughgarden, E. Tardos and V. Vazirani, Eds., Cambridge University Press (2007).

8. Bichler, M., Davenport, A., Hohner, G. and Kalagnanam, J. "Industrial procurement auctions", In Combinatorial Auctions, P. Cramton, Y. Shoham and R. Steinberg, Eds., MIT Press (2010).

9. Hoffman, K.L. "Combinatorial auctions", In Encyclopedia of Operations Research and Management Science, S.I. Gass, M.C. Fu, Eds., pp. 181-192 (2013).

10. Ball, M., Donohue, G. and Hoffman, K. "Auctions for the safe, efficient and equitable allocation of airspace system resources", In Combinatorial Auctions, P. Cramton, Y. Shoham and R. Steinberg, Eds., MIT Press (2010).

11. Caplice, C. and Sheffi, Y. "Combinatorial auctions for truckload transportation", In Combinatorial Auctions, P. Cramton, Y. Shoham and R. Steinberg, Eds., MIT Press (2010).

12. Cantillon, E. and Pesendorfer, M. "Auctioning bus routes: the London experience", In Combinatorial Auctions, P. Cramton, Y. Shoham and R. Steinberg, Eds., MIT Press (2010).

13. Alaei, R. and Ghassemi-Tari, F. "Development of a genetic algorithm for advertising time allocation problems", J. of Ind. and Syst. Eng., 4(4), pp. 245255 (2011).
14. Ghassemi-Tari, F. and Alaei, R. "Scheduling TV commercials using genetic algorithms", Int. J. of Prod. Res., 51(16), pp. 4921-4929 (2013).

15. Cramton, P. "Spectrum auction design", Rev. of Ind. Org., 42(2), pp. 161-190 (2013).

16. Farnia, F., Frayret, J.M., Catherine, B. and Lebel, L. "Time-based combinatorial auction for timber allocation and delivery coordination", For. Policy and Econ., 50, pp. 143-152 (2015).

17. Hohner, G., Rich, J., Ng, E., Reid, G., Davenport, A., Kalagnanam, J., Lee, H. and An, C. "Combinatorial and quantity-discount procurement auctions benefit Mars, Incorporated and its suppliers", Interfaces, 33(1), pp. 23-35 (2003).

18. Metty, T., Harlan, R., Samelson, Q., Moore, T., Morris, T., Sorensen, R., Schneur, A., Raskina, O., Schneur, R., Kanner, J., Potts, K. and Robbins, J. "Reinventing the supplier negotiation process at Motorola", Interfaces, 35(1), pp. 7-23 (2005).

19. Sandholm, T., Levine, D., Concordia, M. and Martyn, P. "Changing the game in strategic sourcing at Procter \& Gamble: Expressive competition enabled by optimization", Interfaces, 36(1), pp. 55-68 (2006).

20. Olivares, M., Weintraub, G., Epstein, R. and Yung, D. "Combinatorial auctions for procurement: an empirical study of the Chilean school meals auction", Manage. Sci., 58(8), pp. 1458-1481 (2012).

21. Cormen, T.H., Leiserson, C.E., Rivest, R.L. and Stein, C., Introduction to Algorithms, 3rd Edition, pp. 10481105, MIT Press (2009).

22. Nisan, N. "Bidding languages for combinatorial auctions", In Combinatorial Auctions, P. Cramton, Y. Shoham and R. Steinberg, Eds., MIT Press (2010).

23. Karp, R.M. "Reducibility among combinatorial problems", In Complexity of Computer Computations, R.E. Miller and J.W. Thatcher, Eds., New York: Plenum (1972).

24. Sandholm, T., Suri, S., Gilpin, A. and Levine, D. "Winner determination in combinatorial auction generalizations", First Int. Joint Conf. on Auton. Agents and Multiagent Syst., 1, ACM, pp. 69-76 (2002).

25. Glover, F. "Scatter search and path relinking", In New Ideas in Optimization, D. Corne, M. Dorigo and F. Glover, Eds., McGraw Hill (1999).

26. Resende, M.G.C., Ribeiro, C.C., Glover, F. and Marti, R. "Scatter search and path-relinking: fundamentals, advances, and applications", In Handbook of Metaheuristics, M. Gendreau and J.Y. Potvin, Eds., Springer (2010). 
27. Alaei, S. and Khoshalhan, F. "A hybrid culturalharmony algorithm for multi-objective supply chain coordination", Scientia Iranica, 22(3), pp. 1227-1241 (2015).

28. Deb, K., Pratap, A., Agarwal, S. and Meyarivan, T. "A fast and elitist multiobjective genetic algorithm: NSGA-II", IEEE Trans. on Evol. Comput., 6(2), pp. 182-197 (2002).

29. Jamshidi, R., Fatemi Ghomi, S.M.T. and Karimi, B. "Multi-objective green supply chain optimization with a new hybrid memetic algorithm using the Taguchi method", Scientia Iranica, 19(6), pp. 18761886 (2012).

30. Jolai, F., Asefi, H., Rabiee, M. and Ramezani, P. "Bi-objective simulated annealing approaches for nowait two-stage flexible flow shop scheduling problem", Scientia Iranica, 20(3), pp. 861-872 (2013).

31. Taguchi, G., Chowdhury, S. and Wu, Y., Taguchi's Quality Engineering Handbook, John Wiley \& Sons (2005).

\section{Appendix}

\section{Generating problem instances}

As mentioned in Section 5, we use a procedure to generate several problem instances for evaluating the performance of our proposed scatter search algorithm in solving the winner determination problem of singleunit combinatorial reverse auction mechanism without free disposal. The procedure in Figure A.1 is used for generating 3 instances for all problem sizes listed in Table 1 (Section 5). Also, the number of decision variables for each generated problem instance is listed in Table A.1.

\section{Biographies}

Reza Alaei obtained BSc degree in Applied Mathematics from University of Tabriz, Tabriz, Iran, and
Table A.1. Different instances of winner determination problem.

\begin{tabular}{ccc}
\hline $\begin{array}{c}\text { Problem size: } \\
(\boldsymbol{m}, \boldsymbol{n})\end{array}$ & Instance \# & Variables \\
\hline I: $(10,25)$ & I-1 & 148 \\
& I-2 & 150 \\
& I-3 & 143 \\
& & \\
II: $(10,50)$ & II-1 & 271 \\
& II-2 & 290 \\
& II-3 & 276 \\
III: $(20,50)$ & III-1 & 290 \\
& III-2 & 293 \\
& III-3 & 293 \\
& & \\
IV: $(20,75)$ & IV-1 & 429 \\
& IV-2 & 421 \\
& IV-3 & 429 \\
\hline
\end{tabular}

MSc degree in Industrial Engineering from Sharif University of Technology, Tehran, Iran. He is currently a candidate of $\mathrm{PhD}$ degree in Industrial Engineering at K.N. Toosi University of Technology, Tehran, Iran. His research interests are mainly focused on combinatorial optimization, supply chain management, and combinatorial auctions. He has authored several papers published in international journals.

Mostafa Setak received BSc, MSc, and $\mathrm{PhD}$ degrees in Industrial Engineering from Sharif University of Technology, University of Science and Technology, and Tarbiat Modares University, respectively, all in Tehran, Iran. He is currently an Associate Professor in Department of Industrial Engineering at K.N. Toosi University of Technology, Tehran, Iran. His research interests include routing problems, supply chain management, and supply chain coordination. He has authored numerous papers presented in conferences and published in national and international journals.

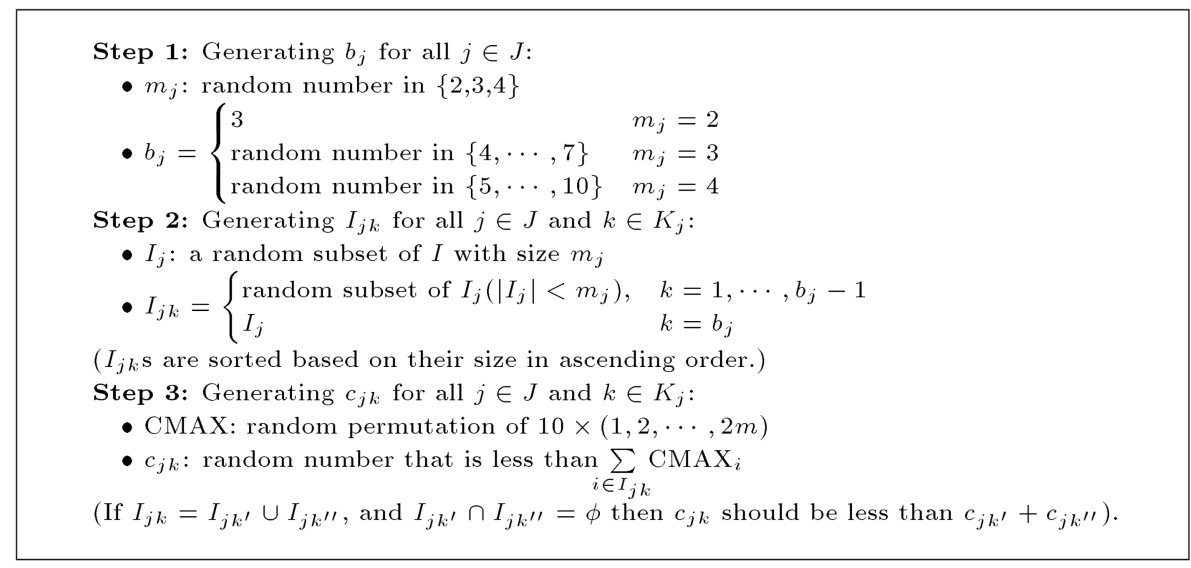

Figure A.1. Procedure of generating problem instances. 